\title{
Lorentz-STEM imaging of Fields and Domains using a High-Speed, High-Dynamic Range Pixel Array Detector at Atomic Resolution
}

Kayla X. Nguyen ${ }^{1}$, Robert Hovden ${ }^{2}$, Mark W. Tate ${ }^{3}$, Prafull Purohit ${ }^{3}$, John Heron ${ }^{3,4}$, Celesta Chang ${ }^{3}$, Sol M. Gruner ${ }^{3}$, David A. Muller ${ }^{2,5}$

${ }^{1}$ Department of Chemistry and Chemical Biology, Cornell University, Ithaca, NY, USA

2.School of Applied and Engineering Physics, Cornell University, Ithaca, NY, USA

${ }^{3 .}$ Department of Physics, Cornell University, Ithaca, NY, USA

${ }^{4}$.Department of Materials Science and Engineering, Cornell University, Ithaca, NY USA

${ }^{5}$ Kavili Institute for Nanoscale Science, Cornell University USA

Renewed interest in Lorentz microscopy of materials has accompanied the arrival of differential phase contrast imaging in scanning transmission electron microscopy (DPC-STEM). Utilizing an annular quadrant detector, small shifts related to the presence of electric or magnetic fields of the scattered beam are inferred from the difference in signal between the vertical or horizontal segments [1,2]. However, once the beam profile is not a top hat function [3], determination of spot shifts remains difficult quantitatively. DPC is also exquisitely sensitive to small changes in crystal tilt [4]. The challenge for quantitative Lorentz-STEM, like holography [5], is to accurately decouple the deflections resulting from crystallographic orientation, local atomic potentials, and the long-range electromagnetic fields.

Here we demonstrate accurate Lorentz-STEM by collecting the entire diffracted beam throughout an image scan using a custom mixed-mode pixel array detector (MMPAD) [6] with exceptional highdynamic range-capable of 1 to $>300,000$ electrons per pixel. At $1 \mathrm{~ms}$ readout and $10 \mathrm{pA}$ probe current at $200 \mathrm{keV}$ in a Tecnai F20, the high-angle scattering is detected with single electron sensitivity (Figure 1a,b) simultaneously with the unsaturated high-intensity central beam and crystallographic Bragg discs (Figure 1a). Thus, changes in the scattered beam's momentum are directly measured over all angles and a specimen's in-plane magnetic and electric fields can be extracted. Importantly, the influence of crystal tilt and absolute local sample thickness variations can also be accounted for. Figure 1c of the xcomponent of the magnetic field in Co imaged by Lorentz-STEM-a system commonly studied by traditional Lorentz-TEM and holography_shows the familiar magnetic ripple texture of the material.

Fast MMPAD readout allows Lorentz-STEM imaging at atomic resolution. Since the entire diffracted beam is recorded, all of the standard STEM imaging modes-e.g. annular dark field (ADF) and bright field - can be specified post hoc [4]. Figure 1a shows an atomic resolution ADF image generated from the MMPAD alongside the mean beam deflections at a domain boundary in the ferroelectric, $\mathrm{BiFeO}_{3}$ showing both long and short range contributions (Fig. 2b,c). Over a larger field, the ferroelectric domains are clearly visible, with sharp boundaries (Figure 3a,b). In both, Fig 2 and 3, the vertical and horizontal components of the deflections the scattered beam are measured.

Naively, these deflections could be converted directly to electric field. However, here the spontaneous polarization of the ferroelectric is accompanied and balanced by a lattice distortion and tilt. The gradient of the potential responsible for the beam deflection is comparably affected by crystal mistilt, thickness, atomic potential [7], and long-range electric fields. Our high-dynamic range PAD offers exciting opportunities for Lorentz-STEM-where crystal orientation, local atomic potentials, and electromagnetic fields can be determined from the full range of low to high-angle electron scattering. [8]

[1] J. Chapman, J. Phys. D. 17, 623 (1984). 
[2] N. Shibata, et al., Nature Physics 8, 611-615 (2012).

[3] Y., Yajima, et al., Bull. Col. Ibaraki Univ. (Nat. Sci) 58, 19-24 (2009) .

[4] T.A. Caswell, et al., Ultramicroscopy 109, 304-311 (2009).

[5] H. Lichte, et al., Ultramicroscopy 93, 199-212 (2002).

[6] W. Vernon, et al., Proc. SPIE 67060U.1-11 (2007).

[7] K. Müller, et al., Nature Communications 5, 1-8 (2014).

[8] Supported by the NSF MRSEC program (DMR 1120296) and the Kavli Institute at Cornell.
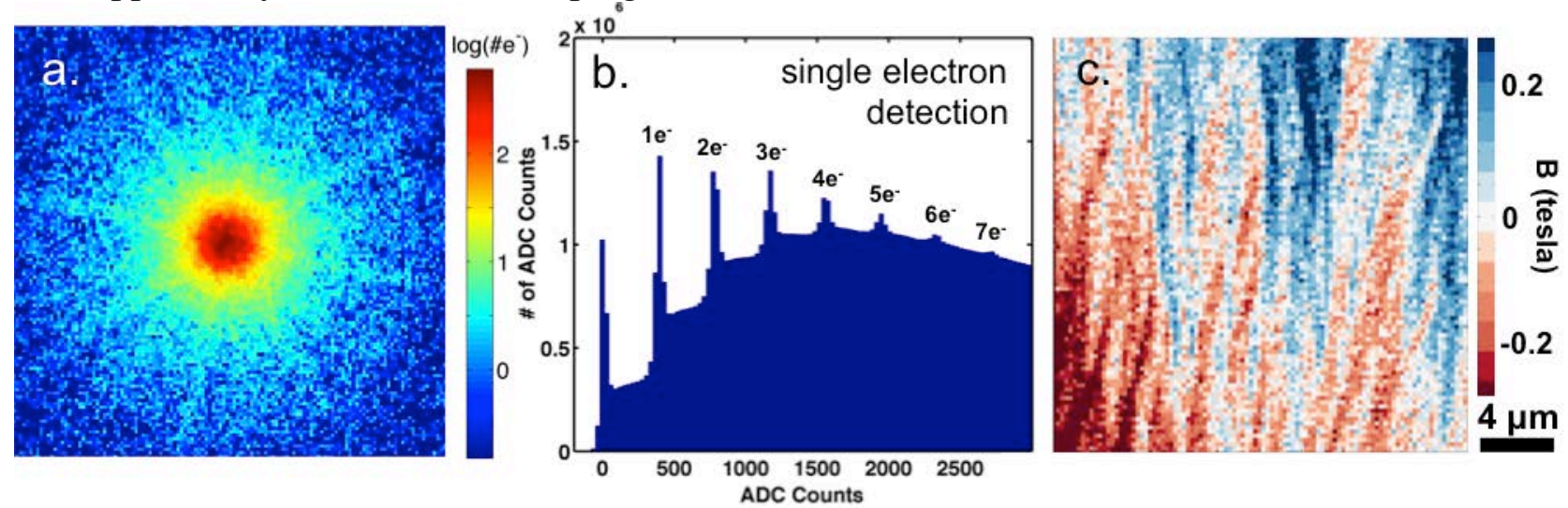

Fig. 1. a) CBED pattern recorded in $1 \mathrm{~ms}$ extracted from a full PAD dataset on $\mathrm{BiFeO}_{3}$. b) Histogram of PAD intensities shows single electron quantization. c) Magnetic field from X-deflections of a Co film.
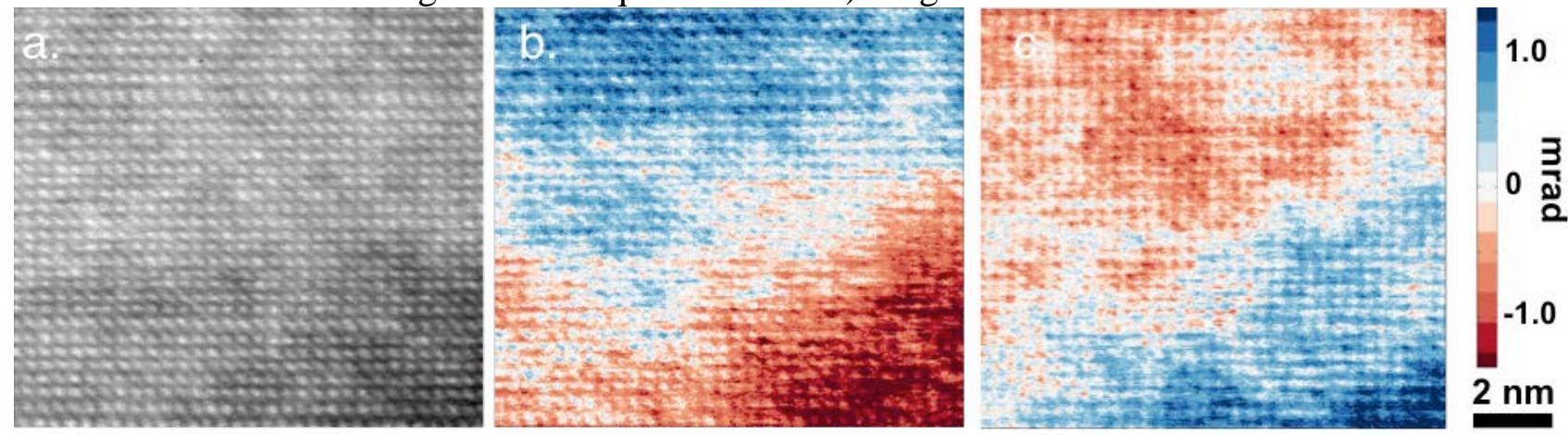

$-1.0$

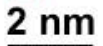

Fig. 2. a) ADF-STEM signal obtained by integration from PAD dataset over 256x256 different beam positions at a $\mathrm{BiFeO}_{3}$ domain boundary. Simultaneous b) $\mathrm{x}$ and c) y components of the beam centroid deflection $\langle\vec{\theta}\rangle$ at atomic resolution. (65 sec acquisition for full PAD datatset).
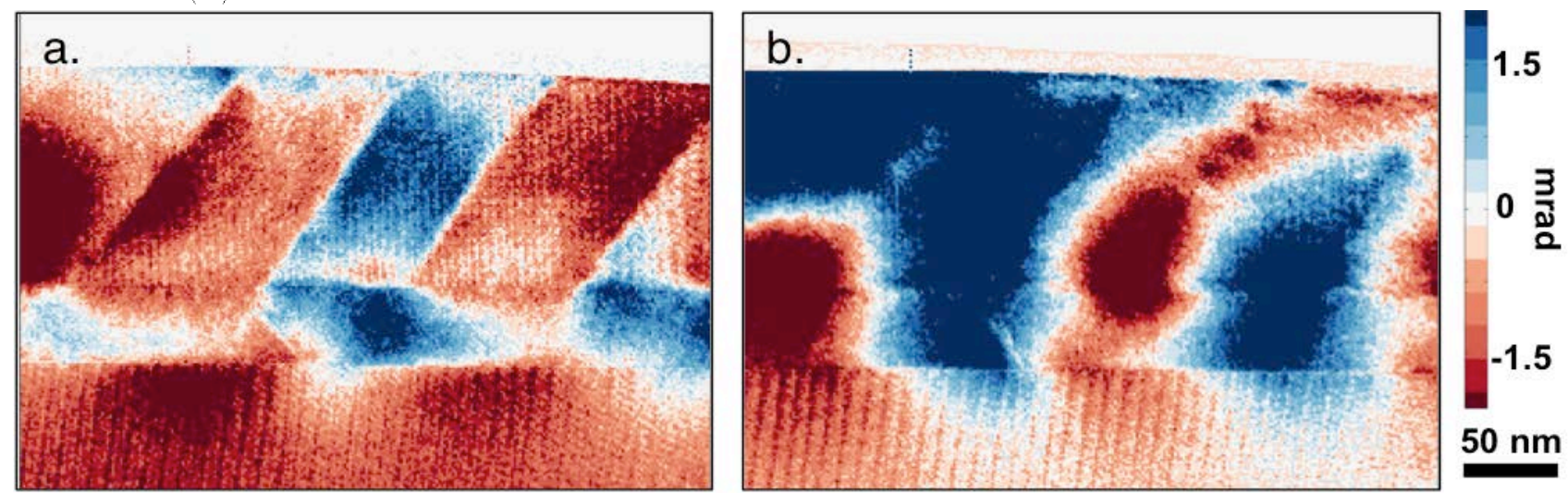

Fig. 3. a) $\mathrm{x}$ and b) y components of the beam centroid deflection $\langle\vec{\theta}\rangle$ show domains and strain in ferroelectric $\mathrm{BiFeO}_{3}$. 